\title{
What Makes News Sharable on Social Media?
}

\author{
XI (CATHY) CHEN ${ }^{1}$ \\ Massachusetts Institute of Technology, USA \\ GORDON PENNYCOOK ${ }^{2}$ \\ University of Regina, Canada \\ DAVID G. RAND ${ }^{3}$ \\ Massachusetts Institute of Technology, USA
}

\begin{abstract}
With the rise of social media, everyone has the potential to be both a consumer and producer of online content. Although one might assume that people share news because they believe it to be true, worldwide concerns about the spread of misinformation suggest that truthfulness may not be a dominant driver of sharing online. Across three studies (total N=3,492), we investigate what content dimensions are associated with social media sharing intentions for a wide range of news headlines. When we examine the relationships between content dimensions using factor analysis, we consistently find separate factors capturing perceived accuracy and evocativeness. The perceived accuracy factor was positively correlated with both sharing intentions and the headline's objective veracity. However, while the evocativeness factor was also positively correlated with sharing intentions, it was consistently negatively correlated with the headline's objective veracity.
\end{abstract}

Keywords: news sharing, social media, accuracy, online decision making, misinformation

${ }^{1}$ Xi (Cathy) Chen: cxic@mit.edu

${ }^{2}$ Gordon Pennycook: gordon.pennycook@uregina.ca

${ }^{3}$ David G. Rand: drand@mit.edu

Date submitted: 2022-11-09. This version: 2023-04-17.

Copyright @ 2023 (Xi (Cathy) Chen, Gordon Pennycook, David G. Rand). Licensed under the Creative Commons Attribution Non-commercial No Derivatives (by-nc-nd). Available at: http://journalqd.org 


\section{Introduction}

The advent of social media has drastically changed the way that people share information (e.g., Lamberton \& Stephen, 2016). One area where this change is particularly salient is news, with more people than ever getting their news from social media (e.g., Pew Research Center, 2017). Traditionally, a small number of news outlets broadcast news to millions of passive consumers. On social media, however, everyone has the potential to be both a consumer and producer of content. Hence, the rise of social media dramatically increases the role word-ofmouth communication plays in news consumption. Among many other things, this dynamic has been argued to have fueled the proliferation of misinformation and "fake news" (Lazer et al., 2018), which has important consequences for a wide range of human decision makings, and major economic impacts. Therefore, in the current work, we investigate how people decide what news (either true or false) to share on social media.

A substantial body of research has examined how features of a piece of content are related to the likelihood of being shared. For example, content that elicits more positive feelings (such as content that is interesting or humorous) is more likely to spread (e.g., Bakshy, et al., 2011; Warren \& Berger, 2011). Beyond simply evoking positive feelings, emotional content that specifically increases arousal (such as anger, anxiety, awe, or surprise) is more likely to be shared (e.g., Berger, 2011; Berger \& Milkman, 2012; AI-Rawi, 2019) and sentiment volatility (i.e., volatility in valence) is suggested to be important in making cultural products more successful (Berger, Kim, \& Meyer, 2021). In addition, moral and emotional content is more likely to be shared (Brady et al. 2017; Brady, Crockett, \& Van Bavel, 2020; Brady, Gantman, \& Van Bavel, 2020) and moral outrage expressions could be amplified by social learning in online social network (Brady, et al., 2021). Furthermore, content that is seen as useful and informative is also more likely to be shared (e.g., Berger \& Milkman, 2012; Heath, Bell, \& Sternberg, 2001).

In the current work, we add to this literature by examining social media sharing intentions of news that varies in objective truth. Although one might assume that people only share news that they believe to be true, in fact empirical evidence suggests that false content may spread as much (Grinberg et al., 2019) or more (Vosoughi, Roy, \& Aral, 2018) than similar true content. Experimental evidence also demonstrates a disconnect between accuracy judgments and sharing intentions: despite saying that accuracy is important to them, people sometimes (even often) share news that they could identify as being false if they considered its accuracy prior to sharing (Pennycook, Epstein, et al., 2021; Epstein, et al., 2021; Pennycook, McPhetres, et al., 2020). 
If accuracy is not a primary driver of choices about what news content to share, then what factor(s) is significantly associated with sharing of accurate versus inaccurate news? From prior work, we can assemble a set of hypotheses. The work reviewed above suggests that emotional evocativeness, informational value, and importance/impact may be associated with news sharing intentions. Furthermore, identity has been suggested to play an important role in sharing (e.g., Brady, Crockett, \& Van Bavel, 2020), such that people are more likely to share news that aligns with their political ideology in both survey experiments (Pennycook, Cannon, \& Rand, 2018; Pennycook \& Rand, 2019; Pennycook, Epstein, et al., 2021) and panel studies on social media (Grinberg et al., 2019). Another line of work suggests that familiarity may be related to sharing, although the direction is unclear: Twitter data shows that novel content is more likely to be shared (Vosoughi, Roy, \& Aral, 2018) whereas survey data suggesting that familiarity is positively associated with sharing (Effron, \& Raj, 2020). Therefore, in the current work, we examine the relationship between sharing intentions and each of these content dimensions, as well as perceived accuracy (i.e., subjective truth) verse headline veracity (i.e., objective truth).

\section{Method}

\section{Participants}

Study 1 was conducted during March 2020 by recruiting American participants from MTurk $\left(N=1,009 ; \mathrm{M}_{\mathrm{age}}=37.1, \%\right.$ Female=43.7). Study 2 was conducted during November 2021 by recruiting American participants from Lucid $\left(N=1,990 ; \mathrm{M}_{\text {age }}=46.4, \%\right.$ Female=51.1 $)$. In Study $2,64.4 \%$ subjects passed the attention check question and completed the study $(N=1,281$; $\mathrm{M}_{\mathrm{age}}=47.2$, \% Female=51.8). Study 3 was conducted during April 2022 by recruiting American participants from Lucid ( $N=1,787 ; \mathrm{M}_{\mathrm{age}}=46.1$, \% Female=50.7). In Study 3, 67.3\% subjects passed the attention check question and completed the study $\left(N=1,202 ; \mathrm{M}_{\text {age }}=47.4, \%\right.$ Female=50.2). For Study 2 and Study 3, the analyses and results presented in the following were based on the subjects who passed the attention check question.

\section{Materials}

\section{Headlines}

Participants in all studies were shown a set of actual headlines from social media (for details of the procedure for selecting headlines, see Pennycook, Binnendyk, Newton, \& Rand, 2021). The headlines were shown along with an image, as it would appear on Facebook. Study 1 
included a set of 216 headlines (72 true, 72 false, and 72 misleading), and each participant rated a random subset of 18 headlines which included all types of news headlines. Study 2 included a set of 200 headlines (100 true and 100 false), and Study 3 included a list of 278 headlines (157 true and 121 false). For Study 2 and Study 3, each participant rated a random subset of 10 headlines which included all types of news headlines. See https://osf.io/q6gwy/ for full list of headlines in each study.

\section{Content Dimensions}

Given the work reviewed in the introduction section, which suggests a variety of links between content dimensions and sharing, we asked participants to rate each headline on various different dimensions (as described below). Dimension questions were all elicited using Likert scales. Last, participants were asked about Sharing ("If you were to see the above article on social media, how likely would you be to share it?").

In Study 1, participants rated Truth ("What is the likelihood that the above headline is true?”), Familiarity (“Are you familiar with the above headline (have you seen or heard about it before?)", Partisanship ("Assuming the above headline is entirely accurate, how favorable would it be to Democrats versus Republicans?"; we constructed a Politically Concordant variable by reversing the scores for participants who were Democrats), Importance ("Assuming the headline is entirely accurate, how important would this news be?"), Emotionality ("How anger provoking is this headline?" and "How anxiety provoking is this headline"?), Humor (“How funny is this headline?"), and Informativeness ("How informative is this headline?").

Study 2 kept the same measures of Truth, Familiarity, Partisanship, and Importance as in Study 1, and had a different set of additional measures: Positivity ("To what extent does this headline make you feel good (happy, excited, hopeful, etc.)?”), Negativity (“To what extent does this headline make you feel bad (angry, sad, worried, etc.)?"), and Sensation ("To what extent is this headline sensationalist and/or exaggerated?").

Study 3 kept the same measures of Truth, Familiarity, Partisanship, and Informativeness as in Study 1, and Sensation as in Study 2, and include two additional measures: Impactful/Important ("How impactful/important is this headline?"), and Surprising ("How surprising is this headline?"). 


\section{Results}

\section{Relationships between Content Dimensions}

We began with assessing how the various content dimensions relate to each other by running Pearson Correlation analyses. We also asked to what extent the different dimensions reflect a smaller number of underlying latent dimensions. To do so, we conducted exploratory factor analysis (EFA), used parallel analysis (Horn, 1965) to determine the number of factors to retain in a principled way, and then used promax rotation to determine loadings.

\section{Study 1}

In Study 1, we had eight content dimensions: "true", "familiar", "political concordant", "important", “anger provoking”, "anxiety provoking", "funny”, and "informative". Figure 1 shows a heatmap for the Pearson Correlation results. Most of the correlations were positive. The correlational coefficients between "anger" and "anxiety", "true" and "informative", "familiar" and "funny", "anxiety" and "informative" were large ( $r$ 's $>0.5)$. Based on these strong correlations, we then used EFA to examine if these dimensions reflect a smaller number of underlying latent structures. 


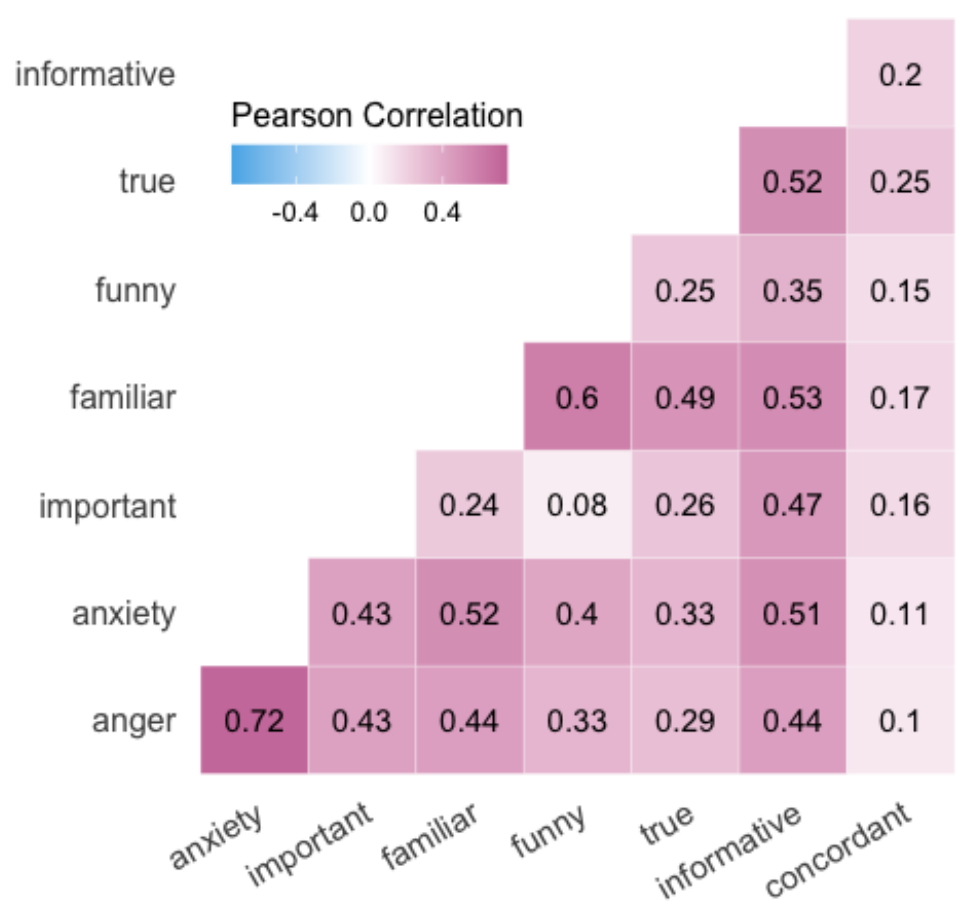

Figure 1. The Pearson Correlation heatmap for the content dimensions in Study $1^{4}$.

Parallel analysis led us to retain three factors from these eight content dimensions. As shown in Table 1, Factor 1 included high loadings on "anger" (0.856), "anxiety" (0.822), “important" (0.473); Factor 2 included high loadings on "true" (0.640), "informative" (0.635), "important" (0.350), and "concordant" (0.325); Factor 3 included high loadings on "familiar" (0.657) and "funny" (0.694). Therefore, Factor 1 seemed to mainly involve dimensions related to emotional evocativeness especially negative feelings; Factor 2 included perceived accuracy, informativeness, and political concordance; Factor 3 was about perceived familiarity and funniness. The results from the EFA suggest perceived accuracy and evocativeness were captured by separate factors.

\footnotetext{
${ }^{4}$ All the correlations were statistically significant, $p<0.05$.
} 
Table 1. Exploratory Factor Analysis with Promax Rotation (Study 1, 3-factor solution) 5 . Loadings above 0.3 shown in bold.

\begin{tabular}{lccc}
\hline Item & Factor 1 & Factor 2 & Factor 3 \\
\hline Anger & $\mathbf{0 . 8 5 6}$ & -0.141 & 0.124 \\
Anxiety & $\mathbf{0 . 8 2 2}$ & -0.100 & 0.212 \\
Important & $\mathbf{0 . 4 7 3}$ & $\mathbf{0 . 3 5 0}$ & -0.269 \\
Familiar & 0.068 & 0.291 & $\mathbf{0 . 6 5 7}$ \\
Funny & 0.059 & 0.008 & $\mathbf{0 . 6 9 4}$ \\
True & -0.050 & $\mathbf{0 . 6 4 0}$ & 0.126 \\
Informative & 0.203 & $\mathbf{0 . 6 3 5}$ & 0.049 \\
Concordant & -0.060 & $\mathbf{0 . 3 2 5}$ & 0.023 \\
\hline
\end{tabular}

In addition, we ran another set of Pearson Correlation analyses between these three factors and found all correlations were positive with high coefficients: $r=0.69$ (between Factor 1 and Factor 2), $r=0.53$ (between Factor 1 and Factor 3), and $r=0.61$ (between Factor 2 and Factor 3).

\section{Study 2}

In Study 2, we had eight content dimensions: "true", "familiar", "political concordant", "important", "boring", "positive", "negative", and "sensational". Figure 2 shows a heatmap for the Pearson Correlation results. Unlike Study 1 where almost all the correlations were significantly positive, the correlations in Study 2 included both positive and negative coefficients. The negative correlations included between "true" and "sensational" ( $r=-0.33)$, and between "important" and "boring" ( $r=-0.29)$. The largest positive correlation was between "positive" and "familiar" ( $r=0.59)$, followed by the correlation between "familiar" and "true" $(r=0.47)$, and then the correlation between "positive" and "true" ( $r=0.39)$. Interestingly, these results show that "true" was positively correlated with "familiar", "positive", etc., while it was negatively correlated with "sensational".

\footnotetext{
${ }^{5}$ In Study 1, the proportions of variance explained by Factor 1, 2, 3 are 21.1\%, 14.4\% and 13.3\%, respectively.
} 


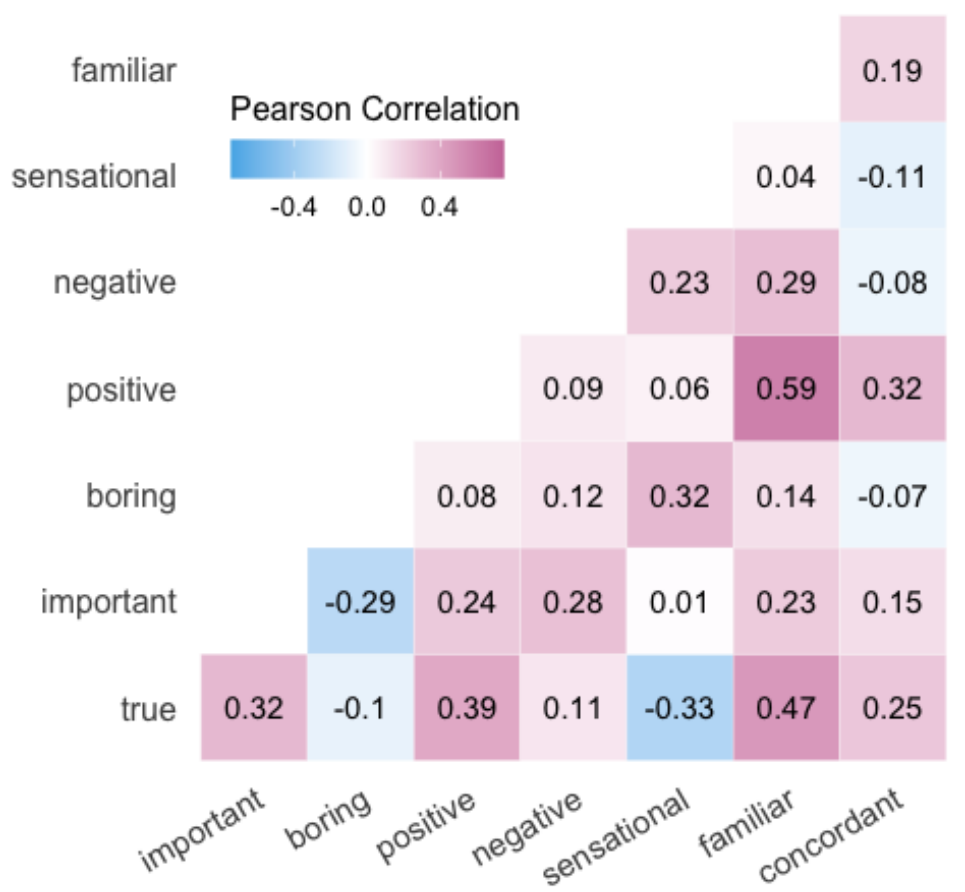

Figure 2. The Pearson Correlation heatmap for the content dimensions in Study $\mathbf{2}^{6}$.

Parallel analysis led us to retain four factors in Study 2. As shown in Table 2, Factor 1 included high loadings on "positive" (0.873), "familiar" (0.594), "concordant" (0.477), and "true" (0.390); Factor 2 included high loadings on "important" (0.991) and "not boring" (-0.371); Factor 3 included high loadings on "sensational" (0.896) and "not true" (-0.408); Factor 4 included high loadings on "negative" (0.647), "familiar" (0.404), and "important" (0.375). Therefore, while one factor (i.e., Factor 1) involved perceived accuracy and other positive dimensions (positivity, familiarity, and concordance), the other two factors focused on perceived importance (i.e., Factor 2) and sensationalism (i.e., Factor 3) respectively, and the last factor was mainly on perceived negativity (i.e., Factor 4). Factors 3 and 4 both tap into the concept of evocativeness. We also check the case with only three factors (See Table A1 in Appendix), and find that the first two factors were very similar to the case with four factors, while the remaining factor had strong weightings for "boring", "negative", and "sensational". Therefore, these results from EFA with either three or four factors show again that perceived accuracy and evocativeness were captured by separate factors.

\footnotetext{
${ }^{6}$ All the correlations were statistically significant, $p<0.05$, except for the one between "sensational" and "important".
} 
Table 2. Exploratory Factor Analysis with Promax

Rotation (Study 2, 4-factor solution) ${ }^{7}$. Loadings above 0.3

shown in bold.

\begin{tabular}{lcccc}
\hline Item & Factor 1 & Factor 2 & Factor 3 & Factor 4 \\
\hline True & $\mathbf{0 . 3 9 0}$ & 0.060 & $\mathbf{- 0 . 4 0 8}$ & 0.228 \\
Important & 0.135 & $\mathbf{0 . 9 9 1}$ & 0.205 & $\mathbf{0 . 3 7 5}$ \\
Boring & 0.085 & $\mathbf{- 0 . 3 7 1}$ & 0.217 & 0.122 \\
Positive & $\mathbf{0 . 8 7 3}$ & 0.001 & 0.188 & -0.058 \\
Negative & -0.113 & 0.141 & 0.081 & $\mathbf{0 . 6 4 7}$ \\
Sensational & 0.089 & 0.101 & $\mathbf{0 . 8 9 6}$ & 0.128 \\
Familiar & $\mathbf{0 . 5 9 4}$ & -0.129 & -0.070 & $\mathbf{0 . 4 0 4}$ \\
Concordant & $\mathbf{0 . 4 7 7}$ & 0.072 & 0.014 & -0.227 \\
\hline
\end{tabular}

We also ran another set of Pearson Correlation analyses between these four factors, in which we found both positive and negative correlations. While Factor 4 was significantly positively correlated with Factor $1(r=0.50)$ and Factor $3(r=0.22)$, Factor 3 was significantly negatively correlated with both Factor $1(r=-0.29)$ and Factor $2(r=-0.42)$.

\section{Study 3}

In Study 3, we had seven content dimensions: "true", "familiar", "political concordant", "important", "informative", "sensational", and "surprising". The Pearson Correlation results show in Figure 3. The positive correlations with noticeably large coefficients were the ones between "true", "important", "informative" ( $r$ 's>0.5), and the one between "sensational" and "surprising" $(r=0.49)$. In addition, consistent with Study 2, "sensational" was again negatively correlated with "true" $(r=-.28)$. Hence, we observed strong correlations among a couple of dimensions as that in the previous two studies, so we next examined the latent structure with ETA.

\footnotetext{
${ }^{7}$ In Study 2, the proportions of variance explained by Factor 1, 2, 3, 4 are 19.2\%, 14.7\%, 13.8\%, and 10.8\% respectively.
} 


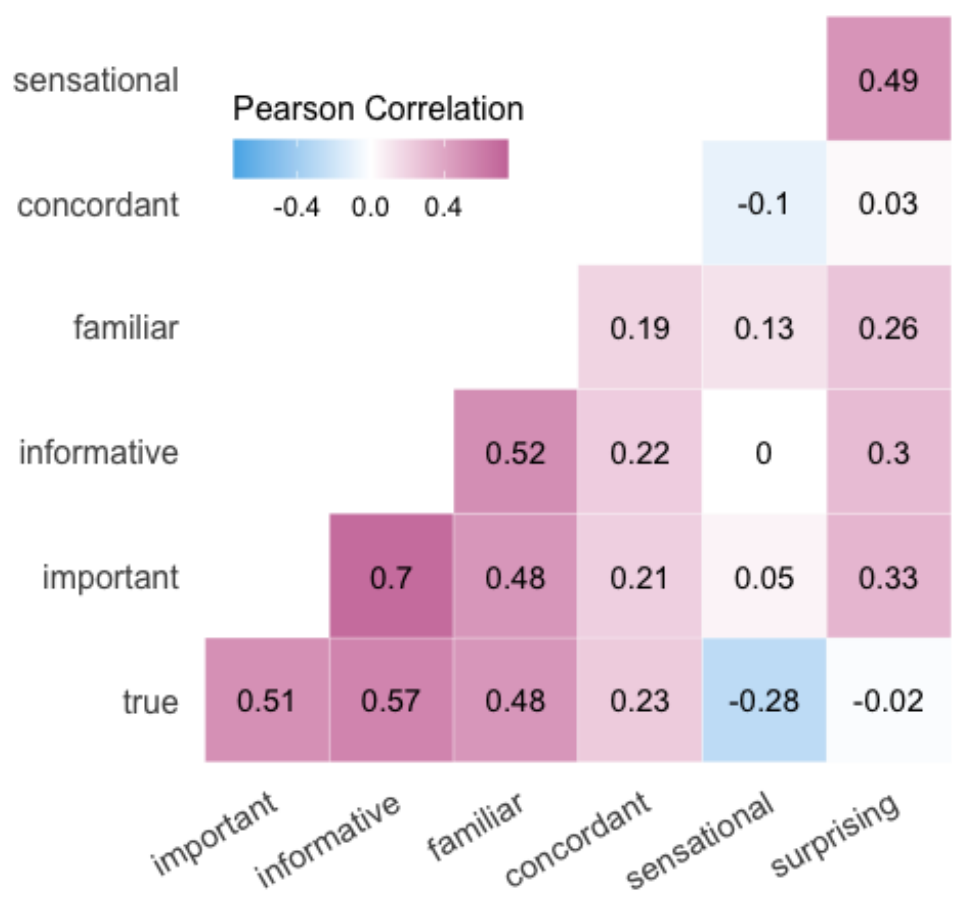

Figure 3. The Pearson Correlation heatmap for the content dimensions in Study $3^{8}$.

Parallel analysis led us to retain three factors in Study 3. As shown in Table 3, Factor 1 included high loadings on "informative" (0.871), "important" (0.831), "true" (0.669); Factor 2 included high loadings on "sensational" (0.737) and "surprising" (0.711); Factor 3 included high loadings on "familiar" (0.930). Therefore, in terms of interpretation, while one factor was mainly about perceived accuracy, informativeness and importance (i.e., Factor 1), the next factor captured evocativeness (i.e., Factor 2), and the last factor was just familiarity (i.e., Factor 3). These results show again that perceived accuracy and evocativeness were captured by separate factors.

\footnotetext{
${ }^{8}$ All the correlations were statistically significant, $p<0.05$, except for the one between "sensational" and "informative".
} 
Table 3. Exploratory Factor Analysis with

Promax Rotation (Study 3, 3-factor solution) ${ }^{9}$.

Loadings above 0.3 shown in bold.

\begin{tabular}{lccc}
\hline Item & Factor 1 & Factor 2 & Factor 3 \\
\hline True & $\mathbf{0 . 6 6 9}$ & -0.266 & 0.115 \\
Important & $\mathbf{0 . 8 3 1}$ & 0.222 & -0.083 \\
Informative & $\mathbf{0 . 8 7 1}$ & 0.161 & -0.050 \\
Familiar & 0.081 & 0.158 & $\mathbf{0 . 9 3 0}$ \\
Concordant & 0.257 & -0.069 & 0.049 \\
Sensational & -0.208 & $\mathbf{0 . 7 3 7}$ & 0.121 \\
Surprising & 0.236 & $\mathbf{0 . 7 1 1}$ & -0.020 \\
\hline
\end{tabular}

Lastly, similar to the previous two studies, we ran another set of Pearson Correlation analyses between these factors: Factor 1 and Factor 3 was positively correlated with a large coefficient $(r=0.63)$, and the other two correlation coefficients were relatively low $(r$ 's<0.1).

\section{Factor Structure Discussion}

Consistently across these studies, we found similar factor structures. First, one factor mainly captured perceived accuracy and positivity (e.g., informativeness and, sometimes but not always, political concordance): Factor 2 in Study 1, Factor 1 in Study 2, and Factor 1 in Study 3 (which we will call the "Accuracy Focused" dimension). Second, one or more factors were related to evocativeness (e.g., anger/anxiety-provoking, negative feelings, sensational, surprising): Factor 1 in Study 1, Factor 3 in Study 2, and Factor 2 in Study 3 (which we will call the "Evocativeness Focused" dimension). Third, one factor included high loadings on perceived familiarity: Factor 3 in Study 1, Factor 4 in Study 2, and Factor 3 in Study 3 (which we will call the "Familiarity Focused" dimension). The one remaining factor, i.e., the Factor 2 in Study 2 (named it as "F2S2" in the following), separated perceived importance from other factors, while in other studies, perceived importance loaded on other factors.

\footnotetext{
${ }^{9}$ In Study 3, the proportions of variance explained by Factor 1, 2, 3 are 29.5\%, 17.5\% and 12.9\%, respectively.
} 


\section{Relationships between Content Dimensions and Headline Veracity}

In the previous section, we assessed how the various content dimensions relate to each other, and we found the consistent existence of several factors of content dimensions unrelated to perceived accuracy (i.e., subjective truth). In the current section, we aim to shed light on the spread of accurate versus inaccurate information - a major focus in current research on social media sharing (Pennycook, \& Rand, 2021; Lazer, et al., 2018) - by examining the relationships between headline veracity (i.e., objective truth; if the news headline is true or false, as determined by fact-checkers) and the content dimensions/factor scores. This set of analyses helps us understand what content dimensions and/or factors are associated with truth discernment - or people's ability to tell true from false. To do so, we ran two sets of OLS regressions predicting headline veracity using either all content dimensions or the factor scores (with one observation per rating, standard errors clustered on participant and headline, and all variables standardized). While the regression with the factor scores provided a more succinct description, the regression with all content dimensions showed the results in more details.

Table 4 summarizes the regressions predicting headline veracity using all content dimensions from the three studies. First, not surprisingly, in all three studies, we observed a strong association between subjective truth ("true") and objective truth (headline veracity): the coefficients on "true" were the highest compared to other content dimensions ${ }^{10}$. Second, "sensational" was negative and significant in predicting headline veracity such that false headlines were judged to be more sensational. Third, a set of evocativeness related dimensions, such as "anger", "anxiety”, "funny", "negative”, "surprising”, were significantly negatively associated with headline veracity. This suggests that misinformation tends to be more evocative than true information. Finally, in two of the three studies, "political concordant" was significantly negatively associated with veracity (which likely reflects imbalance in the political leanings of the headlines and the subject pool).

\footnotetext{
${ }^{10}$ The results from the Wald tests of comparing the differences between coefficients show that the one on "true" was significantly different from the coefficients on all other content dimensions $(p<0.01)$.
} 
Table 4. The OLS regression analyses (standardized) predicting headline veracity using different content dimensions. Standard errors (clustered on participants and headlines) are in parentheses.

\begin{tabular}{|c|c|c|c|}
\hline & & $\begin{array}{l}\text { andent Varial } \\
\text { adline Veraci }\end{array}$ & \\
\hline & Study 1 & Study 2 & Study 3 \\
\hline 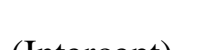 & 0.0000 & 0.0000 & 0.0000 \\
\hline (imtercept) & $(0.061)$ & $(0.063)$ & $(0.052)$ \\
\hline$T_{m}$ & $0.3139 * * *$ & $0.2407 * * *$ & $0.2263 * * *$ \\
\hline 1 rue & $(0.024)$ & $(0.018)$ & $(0.016)$ \\
\hline Impo & -0.0066 & 0.0214 & 0.0184 \\
\hline Important & $(0.021)$ & (0.019) & $(0.018)$ \\
\hline Eomilion & 0.0264 & 0.0270 & $0.0459 * * *$ \\
\hline ramminal & $(0.019)$ & $(0.017)$ & $(0.013)$ \\
\hline Conoordont & -0.0162 & $-0.0258 * *$ & $-0.0220 *$ \\
\hline Concoruamt & $(0.010)$ & $(0.010)$ & $(0.010)$ \\
\hline Informotive & $0.0724 * * *$ & & 0.0276 \\
\hline miormatuve & $(0.017)$ & & $(0.015)$ \\
\hline Sonotion & & $-0.1631 * * *$ & $-0.1793 * * *$ \\
\hline sensallonal & & $(0.012)$ & $(0.013)$ \\
\hline$\Delta$ nor & $-0.0667 * *$ & & \\
\hline Anger & $(0.023)$ & & \\
\hline A pristu & $-0.0412 *$ & & \\
\hline Amxiety & $(0.019)$ & & \\
\hline Funny & $-0.1708 * * *$ & & \\
\hline Funny & $(0.019)$ & & \\
\hline Poring & & $0.0356 * *$ & \\
\hline Bormg & & $(0.013)$ & \\
\hline $\mathbf{N} \quad \mathbf{A}^{\circ}$ & & $-0.0690 * * *$ & \\
\hline Negatıve & & $(0.014)$ & \\
\hline Dociting & & 0.0216 & \\
\hline Posiuve & & $(0.019)$ & \\
\hline Surnrinin & & & $-0.1028 * * *$ \\
\hline sumpnsing & & & $(0.013)$ \\
\hline Observations & 18,162 & 12,810 & 12,020 \\
\hline $\mathrm{R}^{2}$ & 0.116 & 0.121 & 0.153 \\
\hline
\end{tabular}

Note. ${ }^{*} p<0.05, * * p<0.01, * * * p<0.001$ 
Table 5 summarizes the regressions predicting headline veracity using the factor scores from the three studies, and reveals a similar pattern. The Accuracy Focused factor was positively associated with veracity, while the Evocativeness Focused factor was negatively associated with veracity. This suggests again that misinformation may tend to be more evocative than true information. The Familiarity Focused factor was not consistently associated with veracity.

Table 5. The OLS regression analyses (standardized) predicting headline veracity using all the factor scores. Standard errors (clustered on participants and headlines) are in parentheses.

\begin{tabular}{lccc}
\hline & \multicolumn{3}{c}{$\begin{array}{c}\text { Dependent Variable: } \\
\text { Headline Veracity }\end{array}$} \\
& Study 1 & Study 2 & Study 3 \\
\cline { 2 - 4 } (Intercept) & 0.0000 & 0.0000 & 0.0000 \\
F2S1 = F1S2 = F1S3 & $(0.064)$ & $(0.065)$ & $(0.052)$ \\
"Accuracy Focused" & $0.4085^{* * *}$ & $0.1155^{* * *}$ & $0.2319 * * *$ \\
F1S1 = F3S2 = F2S3 & $(0.033)$ & $(0.020)$ & $(0.018)$ \\
"Evocativeness Focused" & $-0.1792^{* * *}$ & $-0.2752 * * *$ & $-0.2978^{* * *}$ \\
F3S1 = F4S2 = F3S3 & $(0.031)$ & $(0.019)$ & $(0.013)$ \\
"Familiarity Focused" & $-0.1542 * * *$ & 0.0235 & $0.0355^{*}$ \\
F2S2 & $(0.023)$ & $(0.022)$ & $(0.015)$ \\
& & -0.0308 & \\
\hline Observations & & $(0.019)$ & 12,020 \\
$\mathrm{R}^{2}$ & 18,162 & 12,810 & 0.147 \\
\hline Note. * $p<0.05, * * p<0.01, * * * p<0.001$ & 0.101 &
\end{tabular}




\section{Relationships between Content Dimensions and Sharing Intentions}

We now examine the relationships between sharing intentions and different aspects of the content (i.e., the content dimensions and factor scores, respectively). Table 6 summarizes the regressions predicting sharing intention using all content dimensions from the three studies. Most of the content dimensions were significantly positively associated with sharing intentions, and the ones that were consistent across the studies included "true", "important", "familiar", "concordant", "informative", and "sensational". Among these dimensions, "familiar" had relatively the largest coefficients ${ }^{11}$.

\footnotetext{
${ }^{11}$ The results from the Wald tests show that the coefficients on "familiar" were significantly different from the coefficients on all other content dimensions ( $p<0.05$; except for the one on "funny" in Study 1).
} 
Table 6. The OLS regression analyses (standardized) predicting sharing intention using different content dimensions. Standard errors (clustered on participants and headlines) are in parentheses.

\begin{tabular}{|c|c|c|c|}
\hline & \multicolumn{3}{|c|}{$\begin{array}{c}\text { Dependent Variable: } \\
\text { Sharing Intention }\end{array}$} \\
\hline & Study 1 & Study 2 & Study 3 \\
\hline \multirow{2}{*}{ (Intercept) } & 0.0000 & 0.0000 & 0.0000 \\
\hline & $(0.013)$ & $(0.014)$ & $(0.014)$ \\
\hline \multirow{2}{*}{ True } & $0.1182 * * *$ & $0.0740 * * *$ & $0.0884 * * *$ \\
\hline & $(0.012)$ & $(0.012)$ & $(0.015)$ \\
\hline \multirow{2}{*}{ Important } & $0.0937 * * *$ & $0.1006^{* * *}$ & $0.1228 * * *$ \\
\hline & $(0.011)$ & $(0.012)$ & $(0.016)$ \\
\hline \multirow{2}{*}{ Familiar } & $0.2398 * * *$ & $0.2320 * * *$ & $0.3326 * * *$ \\
\hline & $(0.018)$ & $(0.017)$ & $(0.017)$ \\
\hline \multirow{2}{*}{ Concordant } & $0.0474 * * *$ & $0.0593 * * *$ & $0.0954 * * *$ \\
\hline & $(0.008)$ & $(0.010)$ & $(0.010)$ \\
\hline \multirow{2}{*}{ Informative } & $0.1853 * * *$ & & $0.1949 * * *$ \\
\hline & $(0.015)$ & & $(0.018)$ \\
\hline \multirow{2}{*}{ Sensational } & & $0.0368 * *$ & $0.0350 * *$ \\
\hline & & $(0.012)$ & $(0.012)$ \\
\hline \multirow{2}{*}{ Anger } & 0.0168 & & \\
\hline & $(0.012)$ & & \\
\hline \multirow{2}{*}{ Anxiety } & $0.1228 * * *$ & & \\
\hline & $(0.014)$ & & \\
\hline \multirow{2}{*}{ Funny } & $0.2478 * * *$ & & \\
\hline & $(0.014)$ & & \\
\hline \multirow{2}{*}{ Boring } & & $-0.0685 * * *$ & \\
\hline & & $(0.011)$ & \\
\hline \multirow{2}{*}{ Negative } & & $0.1332 * * *$ & \\
\hline & & $(0.012)$ & \\
\hline \multirow{2}{*}{ Positive } & & $0.3711^{* * *}$ & \\
\hline & & $(0.014)$ & \\
\hline \multirow{2}{*}{ Surprising } & & & $0.1335 * * *$ \\
\hline & & & $(0.013)$ \\
\hline Observations & 18,162 & 12,810 & 12,020 \\
\hline $\mathrm{R}^{2}$ & 0.574 & 0.462 & 0.484 \\
\hline
\end{tabular}


Table 7 summarizes the regressions predicting sharing intentions using the factor scores from the three studies. Again, the results are consistent with the individual content dimension regressions: All factors were positive and significant in predicting sharing. In terms of decision weights, the Accuracy Focused factor had relatively higher coefficients compared to that of the other factors, whereas the Evocativeness Focused factor had (perhaps surprisingly) the lowest coefficients compared to other factors. We also conducted a series of Wald tests to further examine if each two of the factor coefficients were significantly different from each other. In Study 1, the coefficient on the Evocativeness Focused factor was significantly smaller than the other two factors $(p<0.01)$, which did not significantly differ from each other $(p=0.39)$. In Study 2, the Evocativeness Focused, Accuracy Focused, and Familiarity Focused factors were all significantly different from each other ( $p<0.01$ for all). In Study 3 , the coefficient on the Accuracy Focused factor was significantly larger than the two factors $(p<0.01)$, which did not significantly differ from each other $(p=0.28)$.

Table 7. The OLS regression analyses (standardized) predicting sharing intentions using all the factor scores. Standard errors (clustered on participants and headlines) are in parentheses.

\begin{tabular}{lccc}
\hline & \multicolumn{3}{c}{ Dependent Variable: } \\
& Sharing Intention \\
& 0.0000 & 0.0000 & 0.0000 \\
(Intercept) & $(0.013)$ & $(0.015)$ & $(0.014)$ \\
F2S1 = F1S2 = F1S3 & $0.3457 * * *$ & $0.5744 * * *$ & $0.4665 * * *$ \\
“Accuracy Focused" & $(0.018)$ & $(0.015)$ & $(0.016)$ \\
F1S1 = F3S2 = F2S3 & $0.1463 * * *$ & $0.0794 * * *$ & $0.2102 * * *$ \\
"Evocativeness Focused" & $(0.016)$ & $(0.016)$ & $(0.014)$ \\
F3S1 = F4S2 = F3S3 & $0.3714 * * *$ & $0.1525 * * *$ & $0.2393 * * *$ \\
"Familiarity Focused" & $(0.016)$ & $(0.015)$ & $(0.018)$ \\
& & $0.0836 * *$ & \\
F2S2 & & $(0.013)$ & \\
\hline Observations & 18,162 & 12,810 & 12,020 \\
$\mathrm{R}^{2}$ & 0.562 & 0.442 & 0.476 \\
\hline Note. ${ }^{*} p<0.05, * * p<0.01, * * * p<0.001$ & &
\end{tabular}


Taken together, these results suggest that perceived accuracy may play an important role in what people decide to share on social media, but that other factors - such as evocativeness and familiarity - are also important.

\section{Relationships with Sharing Discernment}

In the previous two sections, we examined the relationships between different aspects of the content and either headline veracity or sharing intention. In the current section, to explore the interplay between these three aspects, we examine how the content dimensions related to sharing discernment - the tendency to share true headlines relative to false news. To do so, we conducted OLS regressions predicting sharing intentions using headline veracity and content dimensions, as well as the interactions between veracity and the content (See results in Table 8, visualized in Figures 4-6). The interaction terms in these analyses allow us to examine how the content dimensions moderate the relationship between headline veracity and sharing intentions - in other words, how the content dimensions relate to sharing discernment.

Two results are most striking. First, across studies, we see that the interaction between familiarity and veracity was negative and significant, suggesting that people are less discerning in their sharing of familiar headlines. Second, in both studies where it was measured, the interaction between headline veracity and "sensational" was significantly positive, suggesting that people are more discerning in their sharing of sensational headlines. Lastly, the interaction terms for "funny", "negative", and "surprising" were also positive and significant in predicting sharing intention, although they were only measured in one study each. 
Table 8. The OLS regression analyses (standardized) predicting sharing intention using headline veracity, content dimensions (except for "true" because it was highly correlated with veracity), and their interactions. Standard errors are clustered on participants and headlines.

\begin{tabular}{|c|c|c|c|}
\hline & \multicolumn{3}{|c|}{$\begin{array}{l}\text { Dependent Variable: } \\
\text { Sharing Intention }\end{array}$} \\
\hline & Study 1 & Study 2 & Study 3 \\
\hline \multirow{2}{*}{ (Intercept) } & 0.0079 & 0.0208 & $0.0400 * *$ \\
\hline & $(0.012)$ & $(0.014)$ & $(0.014)$ \\
\hline \multirow{2}{*}{ Veracity } & $0.0209 * * *$ & $-0.0525 * * *$ & $-0.0313 * * *$ \\
\hline & $(0.006)$ & $(0.008)$ & $(0.008)$ \\
\hline \multirow{2}{*}{ Important } & $0.1006 * * *$ & $0.1237 * * *$ & $0.1452 * * *$ \\
\hline & $(0.011)$ & $(0.012)$ & $(0.016)$ \\
\hline \multirow{2}{*}{ Important $\times$ Veracity } & $0.0382 * * *$ & -0.0045 & 0.0178 \\
\hline & $(0.008)$ & $(0.009)$ & $(0.011)$ \\
\hline \multirow{2}{*}{ Familiar } & $0.2803 * * *$ & $0.2666 * * *$ & $0.3525 * * *$ \\
\hline & $(0.016)$ & $(0.016)$ & $(0.016)$ \\
\hline \multirow{2}{*}{ Familiar $\times$ Veracity } & $-0.0517 * * *$ & $-0.0456 * * *$ & $-0.0645^{* * *}$ \\
\hline & $(0.013)$ & $(0.013)$ & $(0.011)$ \\
\hline \multirow{2}{*}{ Concordant } & $0.0622 * * *$ & $0.0630 * * *$ & $0.0968 * * *$ \\
\hline & $(0.008)$ & $(0.011)$ & $(0.010)$ \\
\hline \multirow{2}{*}{ Concordant $\times$ Veracity } & -0.0112 & 0.0065 & 0.0098 \\
\hline & $(0.006)$ & $(0.009)$ & $(0.007)$ \\
\hline \multirow{2}{*}{ Informative } & $0.2203 * * *$ & & $0.2132 * * *$ \\
\hline & $(0.015)$ & & $(0.017)$ \\
\hline \multirow{2}{*}{ Informative $\times$ Veracity } & -0.0117 & & $-0.0289 *$ \\
\hline & $(0.009)$ & & $(0.013)$ \\
\hline \multirow{2}{*}{ Sensational } & & 0.0017 & 0.0114 \\
\hline & & $(0.012)$ & $(0.012)$ \\
\hline \multirow{2}{*}{ Sensational $\times$ Veracity } & & $0.0577 * * *$ & $0.0725 * * *$ \\
\hline & & $(0.010)$ & $(0.009)$ \\
\hline \multirow{2}{*}{ Anger } & 0.0164 & & \\
\hline & $(0.012)$ & & \\
\hline \multirow{2}{*}{ Anger $\times$ Veracity } & -0.0059 & & \\
\hline & $(0.010)$ & & \\
\hline \multirow{2}{*}{ Anxiety } & $0.1191 * * *$ & & \\
\hline & $(0.014)$ & & \\
\hline \multirow{2}{*}{ Anxiety $\times$ Veracity } & 0.0066 & & \\
\hline & $(0.009)$ & & \\
\hline
\end{tabular}


Funny

Funny $\times$ Veracity

Boring

Boring $\times$ Veracity

Negative

Negative $\times$ Veracity

Positive

Positive $\times$ Veracity

Surprising

Surprising $\times$ Veracity
$0.2432 * * *$

(0.013)

$0.0522 * * *$

(0.010)

$$
\begin{gathered}
-0.0702 * * * \\
(0.011) \\
0.0180 \\
(0.007) \\
0.1282 * * * \\
(0.012) \\
0.0253 * * \\
(0.009) \\
0.3758 * * * \\
(0.014) \\
0.0111 \\
(0.012)
\end{gathered}
$$

\begin{tabular}{lccc}
\hline Observations & 18,162 & 12,810 & 12,020 \\
$\mathrm{R}^{2}$ & 0.568 & 0.467 & 0.493 \\
\hline
\end{tabular}

Note. ${ }^{*} p<0.05, * * p<0.01, * * * p<0.001$ 

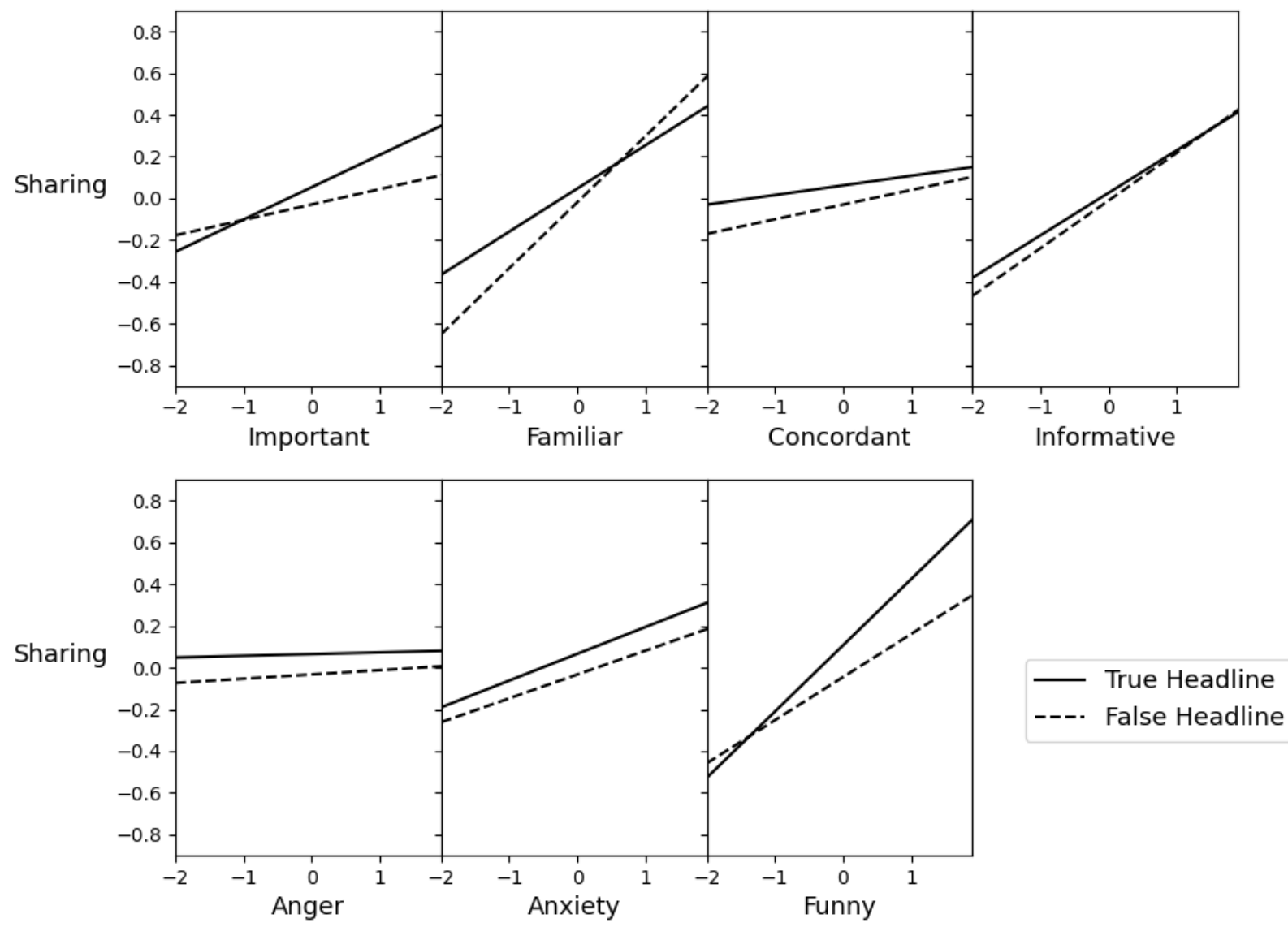

Figure 4. Predicted sharing intentions based on headline veracity and each content dimension (standardized) in Study 1. 

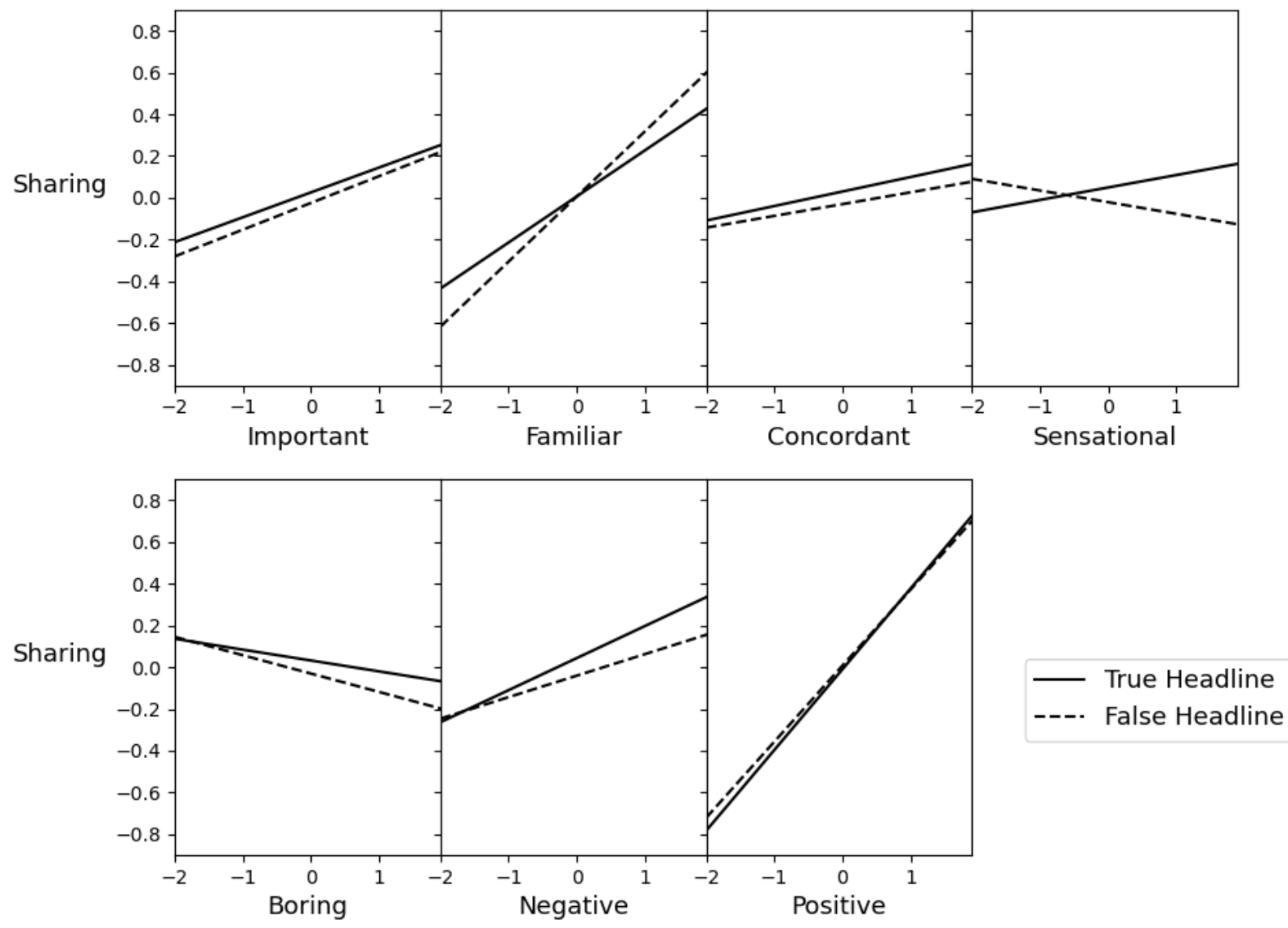

Figure 5. Predicted sharing intentions based on headline veracity and each content dimension (standardized) in Study 2. 

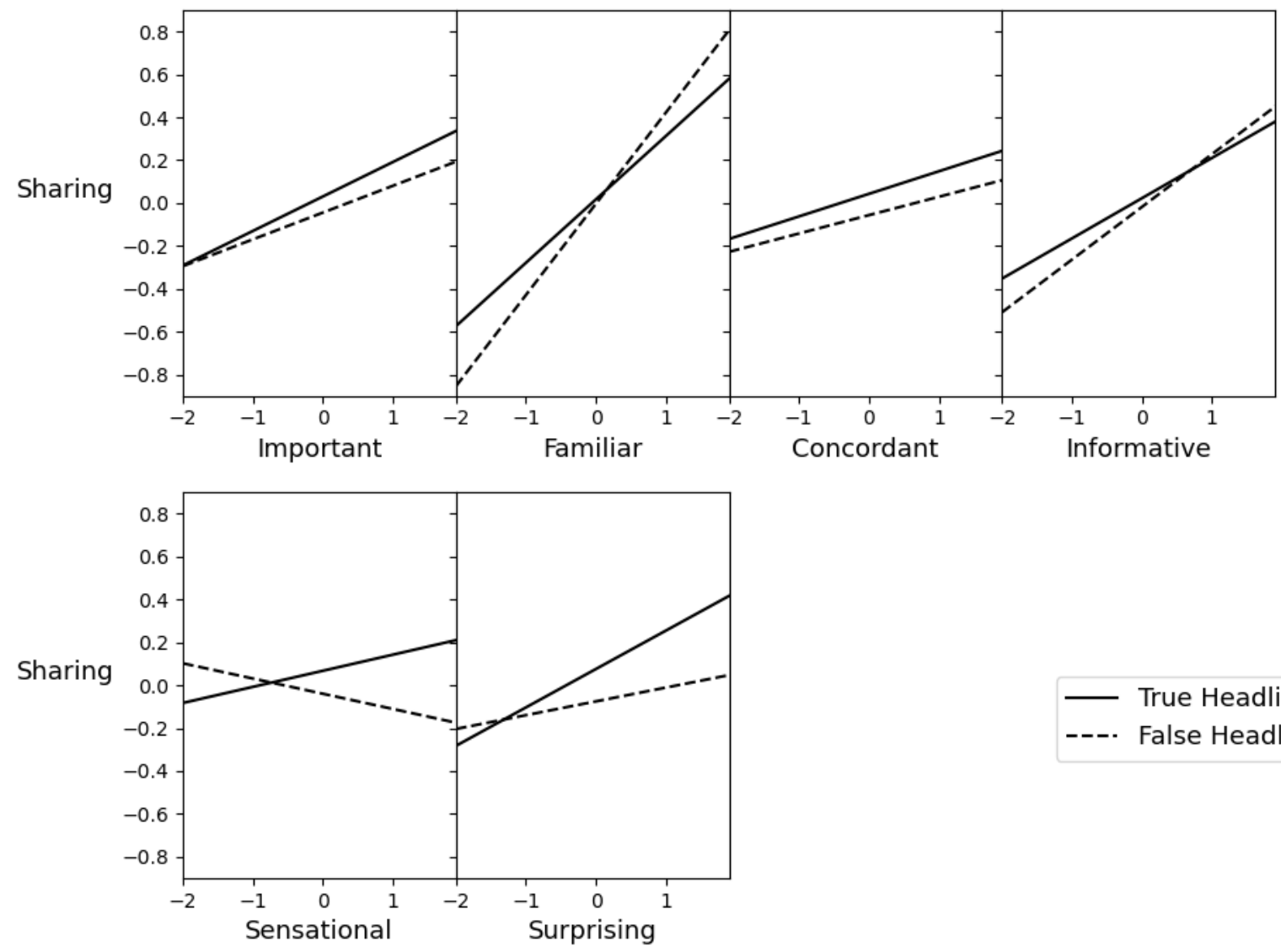

True Headline

---. False Headline

Figure 6. Predicted sharing intentions based on headline veracity and each content dimension (standardized) in Study 3.

\section{General Discussion}

In the current work, we investigated what content dimensions were associated with sharing intentions of true and false news on social media. Across three studies, we found similar factor structures: an Accuracy Focused factor (e.g., truthfulness and informativeness), an Evocativeness Focused factor (e.g., sensational, surprising, anger/anxiety-provoking), and Familiar Focused factor. These factor structures highlight important dimensions beyond perceived accuracy in people's judgments of news headlines.

Furthermore, while perceived accuracy was positively associated with actual objective headline veracity (as determined by professional fact-checkers), evocativeness was negatively associated with objective veracity. This is particularly striking given that both of these dimensions were positively associated with sharing intentions. These results suggest that 
evocativeness may contribute to the sharing of misinformation. Relatedly, when directly examining sharing discernment (i.e., the extent to which people distinguish between true and false headlines when deciding what to share), we found that discernment was lower for familiar and higher for sensational items. Future research is needed to better understand the role of familiarity in one's sharing decisions, such as connecting with the line of "illusory truth effect" research, which has demonstrated that a statement with repeated exposure is more likely to be judged true and accurate than a novel statement (Pennycook, Cannon, \& Rand, 2018; see Dechêne, Stahl, Hansen, \& Wänke, 2010, for a review).

Our findings make several contributions to the existing literature. First, they demonstrate how evocativeness is separate from perceived accuracy and highlight the role that the former may play in one's choice to share inaccurate news online. These results resonate with past research on the spread of online content suggesting that content evoking high-arousal emotions is more viral (e.g., Berger \& Milkman, 2012), extending those results by demonstrating the disconnect between such emotions and objective truth. This disconnect has important implications for understanding the dynamics of news on social media. In particular, our findings shed light on why false content may spread online (e.g., Vosoughi, Roy, \& Aral, 2018). One might imagine that people share misinformation because they purposeful downplay truth. However, our results suggest that it may instead be because people are drawn to content that is evocative and engaging irrespective of its accuracy - and inaccurate content is often more engaging (likely because content creators can create more engaging content if they are not constrained by the truth). This observation can help to inform attempts of social media companies to reduce the spread of misinformation, as well as news organizations that are seeking to generate news that succeeds online. It is also particularly interesting to note that political concordance (despite being a major focus of research on misinformation), did not load heavily on any factor and was less strongly associated with sharing intentions than most other content dimensions. This suggests that the field would do well to put more emphasis on content dimensions beyond politics, even when explicitly examining political news (as we do here).

Finally, there are several limitations of the current work that are important to acknowledge, and various important directions for future research. First, future work should investigate the relationship between sharing, veracity, and other content dimensions not considered here. Second, the current work uses self-reported measures of sharing intentions instead of actual sharing decision data from field experiments. Although there is some reason to believe that self-report sharing intentions are informative (Mosleh, et al., 2020), future research could design field experiments on the social media platforms such as Twitter to improve ecological validity. Third, since the current work focuses on news sharing decisions, future work 
could assess how our results generalize to other online content sharing decisions and contexts such as digital marketing and persuasion. Fourth, Study 1 used a convenience sample from MTurk which makes no effort to be nationally representative, and Studies 2 and 3 used quotamatched samples from Lucid which, although somewhat more representative, have been shown to have substantial issues with inattention. Our focus on descriptive data about variation across headlines, rather variation across subjects, helps to mitigate concerns about the nonrepresentativeness of our samples, and the robustness of our results in Studies 2 and 3 to filtering on attentiveness helps to mitigate concerns about inattention. That being said, it would be useful for future work to attempt to replicate our findings using more representative samples.

\section{Funding Information}

This research was supported by grants from the Social Sciences and Humanities Research Council of Canada, the John Templeton Foundation, and the TDF Foundation.

\section{References}

Al-Rawi, A. (2019). Viral news on social media. Digital journalism, 7(1), 63-79. http://dx.doi.org/10.1080/21670811.2017.1387062

Bakshy, E., Hofman, J. M., Mason, W. A., \& Watts, D. J. (2011, February). Everyone's an influencer: quantifying influence on twitter. In Proceedings of the fourth ACM international conference on Web search and data mining (pp. 65-74). https://doi.org/10.1145/1935826.1935845

Berger, J. (2011). Arousal increases social transmission of information. Psychological science, 22(7), 891-893. https://doi.org/10.1177/0956797611413294

Berger, J., \& Milkman, K. L. (2012). What makes online content viral?. Journal of marketing research, 49(2), 192-205. https://doi.org/10.1509/jmr.10.0353

Berger, J., Kim, Y. D., \& Meyer, R. (2021). What Makes Content Engaging? How Emotional Dynamics Shape Success. Journal of Consumer Research. https://doi.org/10.1093/jcr/ucab010

Brady, W. J., Crockett, M. J., \& Van Bavel, J. J. (2020). The MAD model of moral contagion: The role of motivation, attention, and design in the spread of moralized content online. Perspectives on Psychological Science, 15(4), 978-1010. https://doi.org/10.1177/1745691620917336 
Brady, W. J., Gantman, A. P., \& Van Bavel, J. J. (2020). Attentional capture helps explain why moral and emotional content go viral. Journal of Experimental Psychology: General, 149(4), 746. https://doi.org/10.1037/xge0000673

Brady, W. J., Wills, J. A., Jost, J. T., Tucker, J. A., \& Van Bavel, J. J. (2017). Emotion shapes the diffusion of moralized content in social networks. Proceedings of the National Academy of Sciences, 114(28), 7313-7318. https://doi.org/10.1073/pnas.1618923114

Brady, W. J., McLoughlin, K., Doan, T. N., \& Crockett, M. J. (2021). How social learning amplifies moral outrage expression in online social networks. Science Advances, 7(33), eabe5641. https://doi.org/10.1126/sciadv.abe5641

Dechêne, A., Stahl, C., Hansen, J., \& Wänke, M. (2010). The truth about the truth: A metaanalytic review of the truth effect. Personality and Social Psychology Review, 14(2), 238-257. https://doi.org/10.1177/1088868309352251

Effron, D. A., \& Raj, M. (2020). Misinformation and morality: Encountering fake-news headlines makes them seem less unethical to publish and share. Psychological Science, 31(1), 75-87. https://doi.org/10.1177/0956797619887896

Epstein, Z., Berinsky, A. J., Cole, R., Gully, A., Pennycook, G., \& Rand, D. G. (2021). Developing an accuracy-prompt toolkit to reduce COVID-19 misinformation online. Harvard Kennedy School Misinformation Review. https://hdl.handle.net/1721.1/138124

Grinberg, N., Joseph, K., Friedland, L., Swire-Thompson, B., \& Lazer, D. (2019). Fake news on Twitter during the 2016 US presidential election. Science, 363(6425), 374-378. https://doi.org/10.1126/science.aau2706

Heath, C., Bell, C., \& Sternberg, E. (2001). Emotional selection in memes: the case of urban legends. Journal of personality and social psychology, 81(6), 1028. https://doi.org/10.1037/0022-3514.81.6.1028

Horn, J. L. (1965). A rationale and test for the number of factors in factor analysis. Psychometrika, 30(2), 179-185.

Lamberton, C., \& Stephen, A. T. (2016). A thematic exploration of digital, social media, and mobile marketing: Research evolution from 2000 to 2015 and an agenda for future inquiry. Journal of Marketing, 80(6), 146-172. https://doi.org/10.1509/jm.15.0415

Lazer, D. M., Baum, M. A., Benkler, Y., Berinsky, A. J., Greenhill, K. M., Menczer, F., ... \& Schudson, M. (2018). The science of fake news. Science, 359(6380), 1094-1096. https://doi.org/10.1126/science.aao2998

Mosleh, M., Pennycook, G., \& Rand, D. G. (2020). Self-reported willingness to share political news articles in online surveys correlates with actual sharing on Twitter. Plos one, 15(2), e0228882. https://doi.org/10.1371/journal.pone.0228882

Pennycook, G., Binnendyk, J., Newton, C., \& Rand, D. G. (2021). A practical guide to doing behavioral research on fake news and misinformation. Collabra: Psychology, 7(1), 25293. https://doi.org/10.1525/collabra.25293 
Pennycook, G., Cannon, T. D., \& Rand, D. G. (2018). Prior exposure increases perceived accuracy of fake news. Journal of experimental psychology: general, 147(12), 1865. https://doi.org/10.1037/xge0000465

Pennycook, G., Epstein, Z., Mosleh, M., Arechar, A. A., Eckles, D., \& Rand, D. G. (2021). Shifting attention to accuracy can reduce misinformation online. Nature, 1-6. https://doi.org/10.1038/s41586-021-03344-2

Pennycook, G., McPhetres, J., Zhang, Y., Lu, J. G., \& Rand, D. G. (2020). Fighting COVID-19 Misinformation on Social Media: Experimental Evidence for a Scalable Accuracy-Nudge Intervention. Psychological Science, 0956797620939054. https://doi.org/10.1177/09567976209390

Pennycook, G., \& Rand, D. G. (2019). Lazy, not biased: Susceptibility to partisan fake news is better explained by lack of reasoning than by motivated reasoning. Cognition, 188, 3950. https://doi.org/10.1016/j.cognition.2018.06.011

Pennycook, G., \& Rand, D. G. (2021). The psychology of fake news. Trends in cognitive sciences, 25(5), 388-402. https://doi.org/10.1016/j.tics.2021.02.007

Pew Research Center, September, 2017, "News Use Across Social Media Platforms 2017” https://www.journalism.org/2017/09/07/news-use-across-social-media-platforms2017/pi_17-08-23_socialmediaupdate_0-01/

Vosoughi, S., Roy, D., \& Aral, S. (2018). The spread of true and false news online. Science, 359(6380), 1146-1151. https://doi.org/10.1126/science.aap9559

Warren, C., \& Berger, J. (2011). The influence of humor on sharing. ACR North American Advances. https://www.acrwebsite.org/volumes/1009247/volumes/v39/NA-39

\section{Appendix}

Table A1. Exploratory Factor Analysis with Promax Rotation (Study 2, 3-factor solution). Loadings above 0.3 shown in bold.

\begin{tabular}{lccc}
\hline Item & Factor 1 & Factor 2 & Factor 3 \\
\hline True & $\mathbf{0 . 6 6 3}$ & 0.080 & -0.287 \\
Important & 0.036 & $\mathbf{0 . 9 9 2}$ & 0.185 \\
Boring & 0.116 & $\mathbf{- 0 . 3 6 5}$ & $\mathbf{0 . 3 4 9}$ \\
Positive & $\mathbf{0 . 6 7 5}$ & -0.031 & 0.145 \\
Negative & 0.134 & 0.210 & $\mathbf{0 . 3 5 9}$ \\
Sensational & -0.243 & 0.061 & $\mathbf{0 . 8 3 9}$ \\
Familiar & $\mathbf{0 . 8 3 0}$ & -0.111 & 0.173 \\
Concordant & $\mathbf{0 . 3 2 5}$ & 0.026 & -0.114 \\
\hline
\end{tabular}

